\title{
ANÁliSIS DEL DESPERDICIO DE ALIMENTOS DEL PAE DE DOS SECTORES DE LA CIUDAD DE GUAYAQUIL
}

\author{
Elisa Cedeño $^{a^{*}}$, Erika Loor $^{a}$, Miguel Moreno ${ }^{\mathrm{b}}$
}

${ }^{a}$ Universidad Agraria del Ecuador (Guayaquil, ecedeno@uagraria.edu.ec; ericka18-1@ hotmail.com), ${ }^{b}$ Escuela Superior Politécnica del Litoral(Guayaquil, mamoreno@espol.edu.ec.)

\section{Resumen}

A pesar de las necesidades que existen en diferentes sectores vulnerables y la presencia de iniciativas en alimentación escolar, existe un gran contexto negativo que es el desperdicio de alimentos, el cual ocurre por el comportamiento de los beneficiarios que se condiciona por su cultura, creencias y el desconocimiento de los beneficios que engloba el Programa de Alimentación Escolar (PAE).

Es estudio analizó el desperdicio de alimentos del PAE en dos planteles educativos de la ciudad de Guayaquil, con el propósito de comprender que ocasiona esta problemática. Los resultados demuestran que los factores que inciden al desperdicio son el comportamiento, desconocimiento por parte de representantes, estudiantes sobre los beneficios del programa; además la inasistencia a clases por la pandemia del COVID_19. También existen debilidades en la distribución de los alimentos y ausencia de programas de seguimiento sobre el cumplimiento del PAE.

Los hallazgos identificados evidencian existe desperdicio por lo que imposibilita obtener resultados positivos en cuanto a la nutrición de los educandos y su rendimiento académico. Tampoco existe coordinación para evitar el desperdicio entre aquellos que están directamente relacionados con el programa los servidores públicos que coordinan la entrega de los alimentos, las autoridades del plantel, docentes y padres de familia.

Palabras clave: Desperdicio de alimentos, Programa de Alimentación Escolar (PAE).

\section{Introducción y objetivos}

El entorno infantil requiere de varios cuidados con respecto a la nutrición, por ello es necesario que los ambientes en el cual se desarrollan sean sanos y seguros, para que aprovechen la mayor cantidad de los recursos alimenticios, puesto que los suministros que son desperdiciados causan problemáticas a largo plazo en el presupuesto de las instituciones que financian los programas y lo más importante, los estudiantes no cumplen con los requerimientos nutricionales durante el periodo de clase (Bustamante et al ,2018).

El Programa de Alimentación Escolar (PAE) en Ecuador es un eje importante para el desarrollo del sistema educativo puesto que existe una importante cantidad de estudiantes que no reciben una alimentación adecuada en sus hogares. El programa busca mejorar la alimentación escolar con el fin aportar al estado nutricional y contribuir a un adecuado rendimiento académico a través de la provisión de alimentos a niños, niñas y jóvenes que asisten a las instituciones educativas públicas desde Educación Inicial, Educación General Básica y Bachillerato quienes oscilan entre 3 a 14 años. El PAE consiste en tres modalidades: el desayuno escolar el cual está enfocado en niños de 3 a 5 años, el refrigerio se brinda generalmente a niños y niñas de 3 a 14 años y el almuerzo escolar es direccionado a estudiantes con el servicio de residencia incluyendo a los jóvenes de bachillerato (Ponce y Rosales, 2017).

A pesar de las necesidades que existen en diferentes sectores vulnerables y la presencia de iniciativas en alimentación escolar, existe un gran contexto negativo que es el desperdicio de alimentos, el cual ocurre por el comportamiento de los beneficiados, condicionado por su cultura, creencia y el desconocimiento sobre los beneficios que engloba el PAE por lo que los objetivos del programa no poseen un impacto esperado.

El desperdicio de alimentos es una de las situaciones problemáticas más graves a nivel mundial, donde la desigualdad social y sobrepoblación son los factores más relevantes que afectan la seguridad alimentaria. La Organización de las Naciones Unidas para la Agricultura y Alimentación (FAO) estima que en el mundo una tercera parte de la producción de alimentos se desperdician o se pierden; además, se prevé que para el año 2050 la población mundial oscilará entre 7000 y 9000 millones de personas, motivo por el cual resultará necesario aumentar la producción en un 70\% para cubrir la demanda de alimentos (Bustamante et al.,2018; p. 22)

Según Orbe (2018; pág.67) existen escolares que no consumen todos los productos entregados (galleta y colada) excepto la barra de cereal, esto debido a las características como color, sabor y textura, lo que demuestra una cultura y hábitos inadecuados. 
El desperdicio de alimentos en la institución educativa es significativo diariamente, este no consumo de alimentos incide de manera negativa a la ingesta de nutrientes del estudiante, por tal razón, es necesario considerar acciones que ayuden a optimizar los recursos del PAE a través de pedagogía donde se eduque sobre el cuidado de los bienes públicos que permitan el empoderamiento social para proteger los recursos e infraestructura sino también el acceso al conocimiento y ventajas del correcto uso de los alimentos disponibles en cada ración como beneficio a la formación saludable de niños, niñas y jóvenes para de esa manera erradicar el pensamiento de que la gratuidad es sinónimo de desperdicio (Arévalo, 2016; pág. 82).

Esta investigación analiza el desperdicio de alimentos del PAE en dos planteles educativos Braulia Franco Solís y Homero Espinoza Rendón ubicados en la ciudad de Guayaquil.

\section{Metodología}

La información fue recolectada de fuentes primaria de las dos instituciones educativas seleccionadas y se aplicó dos metodologías: a) encuestas b) entrevistas. La población está compuesta por estudiantes, representantes y personal administrativo un total de 460 y la muestra estratificada por un muestreo no probabilístico fue distribuida de la siguiente manera: 60 representes del nivel inicial (NI), 140 estudiantes de educación general básica (EGB) y 10 docentes o personal administrativo de las instituciones educativas un total de 210 personas.

El mayor número de personas encuestadas fueron los estudiantes quienes son el centro de análisis de la investigación, la consideración de los representantes fue para obtener más información que argumente el proceso investigativo y personas entrevistadas quienes aportaron con datos relevantes sobre el cumplimiento del PAE

Se aplicaron dos encuestas la primera conformada por 5 preguntas dirigida a los estudiantes de EGB y la segunda constituida por 12 preguntas direccionada a los representantes de NI y la entrevista estuvo conformada por 8 preguntas abiertas, dirigidas al personal docente y administrativo de los dos planteles de educación pública.

\section{Resultados}

\subsection{Determinar el funcionamiento del Programa de Alimentación Escolar en Ecuador}

El proceso para cumplir con el programa de alimentación posee los siguientes pasos: La Unidad Operativa de Colación Escolar recibe la nómina de estudiantes matriculados en los establecimientos educativos y en función de la demanda, determina el número de productos alimenticios a distribuir. Luego interviene el Ministerio de Educación quien es el encargado de la logística de distribución; mismo que entregaba de manera mensual los alimentos en cada institución educativa beneficiaria.

Las autoridades educativas y/o delegados son los responsables de recibir las raciones alimenticias y verificar las cantidades, caducidad y la calidad de los productos recibidos, visualizando la textura y apariencia en general la entrega a los estudiantes debe ser diariamente para su consumo en el interior de las Instituciones Educativas a primeras horas de la mañana o en el primer receso.

En la crisis sanitaria el cumplimiento del PAE presentó serios inconvenientes y el proceso normal para entregar las raciones alimenticias sufrió importantes cambios: la colación fue entregada una vez al mes y distribuida a los padres de familia en un día indicado por las autoridades de las instituciones educativas.

Para los representantes de los niños del nivel inicial los alimentos del PAE generan un gran aporte a la salud y nutrición del estudiante pero un segmento importante consideró que no, lo cual influye en el desperdicio de los alimentos que puede ser debido a una débil socialización de la importancia alimenticia en la etapa escolar.

La entrega de las raciones se realizaba en una sola jornada al ingresar a la escuela y normalmente recibían dos productos: leche y galletas, algunas ocasiones un producto: leche, jugo, barra nutritiva o galletas. En contadas ocasiones 3 o 4 productos. Por lo que no existía distribución adecuada de los productos lo cual genera desabastecimiento, menor número de beneficiados y elevadas probabilidades de desperdicios.

\subsection{Identificar de los factores que inciden en el desperdicio de los alimentos del PAE}

Los principales factores identificados que inciden en el desperdicio de alimentos del PAE en las dos instituciones educativas se debe principalmente a la inasistencia de los alumnos en los planes educativos por la crisis sanitaria generada por el COVID 19, los gustos, preferencias, exceso de productos recibidos por inadecuado pedido, escaso control de las instituciones educativas y de los padres de familias, los cuales son elementos que influyen de manera directa en el desaprovechamiento de las raciones alimenticias. El desconocimiento también se perfila como un factor de riesgo que incita al aumento de los desperdicios del PAE, debido a que los padres soslayan la importancia de promover en el infante una cultura alimenticia orientada al beneficio de la salud y rendimiento escolar 
Los alumnos del nivel inicial no consumían todos los productos debido al nivel de aceptación de sabores siendo los jugos procesados el de menor satisfacción y la mayoría de los alimentos no ingeridos son consumidos por el resto de la familia, los regalan e incluso se caduca y son desechados.

La mayoría de los estudiantes de Educación General Básica sí consumen los alimentos que provee el PAE, pero un número importante no, el $55 \%$ que consumen los productos alimenticios presentaron un comportamiento poco estable en cuanto a la frecuencia de consumo, quienes lo ingerían solo de 1 a 2 veces por semana.

La distribución de los productos alimenticios que PAE se daba al ingresar a la institución muchos ya han desayunado en casa y ya no los consumía sino que los llevaban a sus hogares lo cual es un entorno que crea las condiciones para la presencia del problema social de desperdicio de alimentos.

Cuando se consultó a los estudiantes de Educación General Básica si consumían los productos alimenticios del PAE, el 55\% mencionaron que no por el bajo nivel de satisfacción de los productos.

La mayoría de los estudiantes desconocen los beneficios que poseen los alimentos evidencia un bajo nivel de conciencia sobre la importancia de ingerir alimentos con gran aporte nutritivo que favorecen al desarrollo cognitivo del educando.

\subsection{Determinación de la cantidad de alimentos que se desperdicia en el PAE de los dos sectores de la ciudad de Guayaquil.}

El funcionamiento del PAE empieza con la explicación del programa, con la intención de aprovechar los recursos alimenticios brindados por el Estado. Las raciones alimenticias son entregadas a las instituciones educativas de acuerdo con la cantidad de estudiantes que estas posean y se pondera el hecho de que cuentan un alto contenido nutricional que contribuye con el fortalecimiento de la salud en los estudiantes. Por efecto de la crisis sanitaria la entrega de alimentos del PAE se realiza una vez por mes, donde son citados los padres de familia en un día particular para receptar las raciones alimenticias

Para la ejecución del PAE existe la articulación de diversas instituciones estatales que coordinan esfuerzos para que los alimentos lleguen a las instituciones educativas siendo el principal responsable del programa es el Ministerio de Educación a pesar de que la cantidad de alimentos que es suministrada alcanza para la demanda de estudiantes hay deficiencias en la coordinación lo que provoca falta de provisión efectiva de las raciones. En las dos instituciones los docentes y personal administrativo manifestaron que existe desperdicio de alimentos en el PAE que intentan juntar esfuerzos para que esto no suceda pero no ha sido suficiente.

Los factores predominantes del desperdicio de alimentos es el ausentismo de los estudiantes e inasistencias por efectos de la COVID 19 y un escaso control por las autoridades y padres de familia en la distribución de las raciones. No existe una cuantificación exacta de la cantidad que de desperdicio en las instituciones educativas sin embargo lo relevante es que reconocen que existe el desperdicio y realizan esfuerzos para darle un tratamiento en el que los alimentos no lleguen a desperdiciarse pero, necesitan ser reforzados con planes estratégicos en donde se establezcan mecanismos para brindar un tratamiento adecuado al excedente de alimentos que no son utilizados.

\section{Conclusiones}

El inicio de la investigación fue antes de la pandemia del Covid-19, pero el proceso investigativo culminó durante la crisis sanitaria, lo cual no permitió completar de forma correcta la investigación de campo y no se pudo cumplir con la cobertura investigativa de todas las unidades de observación establecidas.

Los principales factores identificados que inciden en el desperdicio de alimentos del PAE en las dos instituciones educativas es la inasistencia de los alumnos además los gustos, preferencias y el exceso de productos recibidos por inadecuado pedido elementos que influyen de manera directa en el desaprovechamiento de las raciones alimenticias, siendo tiradas a la basura y contaminando el medio ambiente, además repercuten en el cumplimiento del PAE exponiendo a las instituciones educativas a perder el beneficio de la alimentación escolar por la inadecuada gestión durante el abastecimiento y la distribución del alimento.

La cantidad de alimentos que se desperdicia del PAE en las instituciones educativas de los dos sectores de Guayaquil son en diferente proporciones según la perspectiva de la Escuela Braulia Franco Solís se desecha un porcentaje estimado del 15\% cuando no logran una distribución adecuada de las colaciones o al no encontrar personas a quien donar los productos sobrantes, mientras que la Escuela Homero Espinoza Rendón dice que el desperdicio es en cantidades mínimas a pesar de los factores influyentes para dicha situación, lo puede indicar una gestión más efectiva en cuanto a la distribución de las raciones. 


\section{Bibliografía}

Arévalo, O. (2016). Análisis del desperdicio de alimentos en el almuerzo escolar del Colegio Distrital Ciudadela Educativa, una mirada desde las dimensiones de la Seguridad Alimentaria y Nutricional. (Tesis de Posgrado). Universidad Nacional de Colombia, Bogotá.

Bustamente, M., Afonso, A., \& Ríos, I. D. (2018). Análisis Exploratorio del Desperdicio de Alimentos en Plato en Comedores Escolares en España. Revista Ciencias de la Vida, 28(2), 20-42.

Orbe, D. (2018). Consumo y aceptación de los alimentos del Programa de Alimentación Escolar en los preescolares en la Unidad Educativa Tulcán. (Tesis de Grado). Universidad Técnica del Norte, Ibarra.

Ponce, J., \& Rosales, C. (2017). Evaluación de impacto del programa de alimentación escolar. Quito: Dirección de investigaciones. 